\title{
A modelagem digital do timbre da viola caipira como fundamento para a composição de Bestiário III
}

The digital modeling of the "viola caipira" tone as a basis for the composition of Bestiary III

Rodolfo Coelho de Souza Universidade de São Paulo rcoelho@usp.br

Guilherme Henrique Santos da Costa Universidade de São Paulo guilherme.santos.costa@usp.br 


\section{Resumo}

Numa perspectiva parcial, este é um relato de experimentos que visa a produzir a síntese de sons semelhantes aos da viola caipira, empregando variações do método Karplus-Strong. Esse método de síntese sonora baseia-se em filas de atraso (delay-lines) e implementa o modelo de guias de onda (waveguides). Nesse sentido trata-se de um estudo de modelagem física no sentido clássico. As variantes desenvolvidas tiveram como ponto de partida os algoritmos de Lazzarini et al. (2016) e Mikelson (2000). Numa perspectiva ampliada, o estudo buscou incorporar ao modelo simulações de outras características acústicas do instrumento, como o choque de cordas duplas, os componentes modais, os formantes e a ressonância do corpo do instrumento. $O$ algoritmo híbrido resultante incorporou uma variedade de outros métodos, como a síntese por soma de ondas senoidais e resíduo de ruído de Serra (1997) e a técnica FOF descrita por Clarke (2000). A pesquisa de Paiva (2017) forneceu os valores experimentais usados na parametrização desse modelo. Os resultados foram aplicados na composição de Bestiário III para violão e sons eletrônicos gerados com esse modelo da viola caipira. A matriz de alturas e as proporções de complexos rítmicos foram livremente inspiradas na análise espectral da viola por Paiva (2017).

Palavras-chave: Viola Caipira; Modelagem Física; Csound; Síntese Karplus-Strong; Música Eletroacústica Mista.

\section{Abstract}

In a partial perspective, this is a report about experiments that aimed to produce the synthesis of sounds similar to a Brazilian folk guitar, using variations of the Karplus-Strong method. Delay-lines implementing the waveguide model are the basis of this sound synthesis method. Therefore, this study belongs to the field of physical modelling. The departing point of the developed variants were the algorithms of Lazzarini et al. (2016) e Mikelson (2000). In a broader perspective, this study searches to incorporate to the model other characteristics of the acoustic instrument, as the double strings strikes, modal components, formants and the resonance of the instrument's body. The resulting hybrid algorithm incorporated a variety of other methods, as the synthesis by sum of sine waves plus noise of Serra (1997) and the FOF technique described by Clarke (2000). The research of Paiva (2017) provided experimental values used in the model parametrization. The results were used to compose the piece Bestiário III for guitar and electronic sounds generated after the viola caipira model. The pitch matrix and the proportions of the complex rhythms were loosely inspired by Paiva's (2017) spectral analysis of the viola caipira.

Keywords: Brazilian Folk Guitar; Physical Modeling; Csound; Karplus-Strong Synthesis; Mixed Eletroacoustic Music. 


\section{Uma encruzilhada na análise da música contemporânea}

Ao me propor a comentar uma composição que eu mesmo escrevi, seria estranho apresentar uma análise convencional da própria obra, visto que um compositor dispõe das informações de bastidor de como ela foi gerada. Isso tornaria um jogo de cartas marcadas uma análise que tentasse relatar o processo composicional do ponto de uma engenharia reversa, isto é, de como se deu o processo gerativo da obra. Por outro lado, após o serialismo de Boulez, e ainda as propostas estocásticas de Xenakis e aleatórias de Cage, ou mesmo a maioria de outras técnicas composicionais da segunda metade do século XX que não se baseiam em lógicas lineares de derivação dos materiais, tornou- se impossível conceber a análise musical como a retroversão da geração dos materiais empregados na obra. Uma análise, em sentido clássico, como por exemplo a contagem da série em uma peça dodecafônica, passou a ser uma missão impossível, anão ser que o compositor contrabandeie por baixo da mesa as chaves de seu processo composicional. Essa tendência, que se generalizou nas últimas décadas, de que cada compositor desenvolva seu idioleto de técnicas, apresenta um sério obstáculo para a continuidade da análise musical no seu sentido histórico

Keller e Ferneyhough (2004) enfrentaram esse problema por meio de uma proposição original e fecunda. Se não é possível através da análise retraçar a gênese de uma obra musical, pode-se alternativamente construir um modelo paralelo que a simule e nos permita compreendê-la melhor. Se essa modelagem reproduzir convincentemente os resultados perceptivos da obra, teremos obtido um bom caminho alternativo para entender sua gênese.

Esse método é particularmente apropriado para as obras recentes que partem de uma experimentação sobre a própria natureza dos sons sobre os quais trabalha um compositor. Assim, torna-se possível analisar peças escritas com técnicas espectralistas, como as de Grisey, ou mesmo as estocásticas instrumentais: aliás, não por acaso, como a peça de Xenakis que Keller e Ferneyhough analisaram em seu artigo seminal.

A análise musical [...] funciona suficientemente bem com peças determinísticas, mas não tão bem na discussão de obras baseadas em "forma processo". ST/10-1 080262 (XENAKIS,1967), por se tratar de uma peça algorítmica, pode ser vista como uma entre muitas possibilidades de realização de um modelo de composição particular. Portanto, entender os mecanismos do modelo torna-se um pré-requisito para a compreensão da peça. Sugerimos que a especificação de um modelo para aspectos salientes da peça - uma análise por modelagem - pode fornecer uma abordagem metodologicamente viável. (KELLER; FERNEYHOUG, 2004, p.161). ${ }^{1}$

\footnotetext{
$1 \quad$ Musical analysis [...] functions sufficiently well with deterministic pieces but is less well suited to the discussion of process based on musical phenomena. ST/10-1 080262 (XENAKIS, 1967), as an algorithmic

piece, can be viewed as one among the many possible realizations of a particular compositional model. Hence, to understand the mechanisms of the model is a prerequisite to understand the piece. We suggest that the specification of a model for salient aspects of the piece - analysis by modeling - may provide a methodologically viable approach (KELLER; FERNEYHOUGH, 2004, p.161).
} 
Por se tratar de uma modelagem, e não de uma análise tradicional, desaparece o constrangimento para que ela seja apresentada pelo próprio compositor. É certo que o compositor detém a priori as informações dessa modelagem, mas é certo também que ele tem o direito de autor dessas informações, que, por outro lado, seriam difíceis de se deduzir por outras vias. Isso torna eticamente justificado o produto acadêmico que resulta de uma apresentação do modelo.

\section{A proposta de Bestiário III}

Esta peça pertence a um ciclo de seis composições escritas para instrumentos e sons eletrônicos. Cada uma delas tem como referência extramusical algum dos animais fantásticos que seduziram a imaginação dos primeiros viajantes pelos territórios do novo mundo durante o período colonial que neles viam a materialização dos monstros descritos nos bestiários medievais. No caso do Bestiário III, o bicho é o urutaurana, uma espécie de gavião brasileiro que porta um penacho na cabeça e que era desconhecida dos primeiros europeus que aqui aportaram. $O$ grito agudo e ameaçador dessa ave que caça pequenos mamíferos no solo funcionou como motivo sugestivo para a composição. ${ }^{2}$

Como em todas as outras peças do ciclo, esse animal da fábula é representado por meio da associação ao timbre de um instrumento cuja sonoridade será modelada e expandida por meios digitais. Neste caso o instrumento tocado ao vivo é o violão, mas a parte eletrônica baseia-se na modelagem da viola caipira, também conhecida em períodos históricos anteriores como viola de arame. ${ }^{3}$

Embora o violão e a viola caipira pertençam à mesma família de instrumentos agrupados por Galpin em 1937 na categoria organológica dos cordofones beliscados (ver HENRIQUE, 2004), a sonoridade de cada um deles tem diversas características distintas. Para nossa pesquisa salientamos três diferenças principais: o violão moderno usa cordas de náilon enquanto a viola caipira usa cordas metálicas; as cordas do violão são únicas enquanto as da viola caipira são duplas; a caixa de ressonância do violão moderno é maior e com formato diferente da caixa da viola caipira. Cada uma dessas características idiomáticas justificou a exploração de variantes de timbre na modelagem digital.

Além da geração de sons eletrônicos modelados pela viola caipira, a composição da parte instrumental de Bestiário III utilizou livremente o princípio da escola espectralista ao mapear uma matriz de alturas e as proporções das durações a alguns resultados da análise espectral do instrumento (conforme PAIVA, 2017).

\footnotetext{
2 Para se conhecer um pouco dessa espécie, ver: https://www.youtube.com/watch?v=8pAEcmfla6w

3 Para se conhecer em detalhes as características de luteria do instrumento, vide Corrêa (2014).
} 


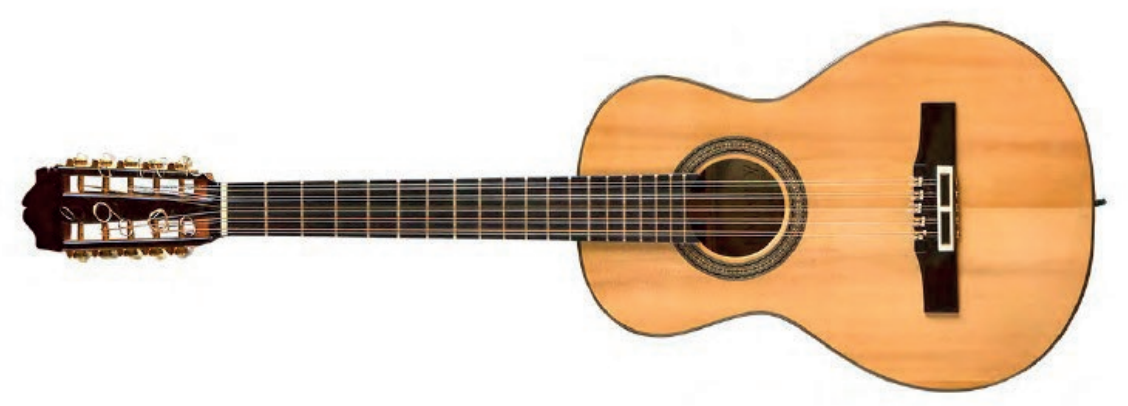

Fig. 1: Viola caipira moderna, fabricada em 2003

(extraído de CORRÊA, 2014, p.82)

\section{Sobre o uso do conceito de modelagem física neste projeto}

A sonoridade dos instrumentos usados na música tradicional brasileira, nas diversas bases culturais que a formaram, constitui um repositório muito rico de possibilidades para investigação e inspiração para o trabalho criativo. Quando nos distanciamos do país e somos confrontados com paradigmas de outras culturas é possível perceber com mais clareza quais são as características particulares que nos diferenciam e formam nossa identidade, inclusive na sonoridade das coisas que nos cercam. Não se trata apenas de recorrer ao elemento exótico como recurso para marcar nossa diferença, ainda que inevitavelmente possamos estar sujeitos a essa leitura. Trata-se principalmente de reconhecer que é mais eficiente e gratificante debruçar-se sobre objetos familiares e significativos para nossa cultura. A viola caipira encaixa-se nesse paradigma. A Universidade de São Paulo, instituição em que os autores desta pesquisa trabalham, foi pioneira na inclusão do ensino da viola caipira em seu currículo, o que também colaborou para inspirar este trabalho.

Em obras anteriores, como no Concerto para Computador e Orquestra (2000), muitos sons eletrônicos foram gerados em Csound, tomando como base transformações de gravações de instrumentos tradicionais brasileiros, como o berimbau, a cuíca, chocalhos e zunidores (COELHO DE SOUZA, 2005). Em outra obra, O Livro dos Sons (2010), a kalimba da Bahia serviu para uma modelagem usada para a geração dos sons eletrônicos empregados na obra (COELHO DE SOUZA, 2011). A série dos Bestiários dá continuidade a esse projeto de longo prazo.

Este projeto pertence, portanto, à área da modelagem física. É necessário, todavia, esclarecer o que se entende aqui por modelagem física. Num âmbito restrito, o conceito de modelagem física é definido como a aplicação do processo de síntese por waveguide à modelagem de instrumentos reais (Loy, 2007, p.439). Todavia, alguns autores, como o próprio Loy (2007), e ainda De Poli (1998) e Xavier Serra (1997), entendem que o campo de pesquisa da modelagem física é amplo e abrangente. Loy (2007, p.433) amplia a definição de modelagem física, considerando-a como "o estudo das interações causais dos sistemas vibratórios que são o fundamento dos sons naturais. Isto inclui o modo como a energia viaja através do executante, para e através do instrumento musical, como suas ressonâncias afetam a vibração resultante e como essas vibrações se 
propagam no ar circundante". Portanto, o conceito não estaria restrito ao uso de waveguide e sua síntese através de filas de atraso (delay lines). Serra (1997, p.90) propõe uma abordagem alternativa, que chama de "modelo espectral". Ela consiste numa combinação de síntese aditiva, replicando os parciais senoidais dos sons naturais, somada aos componentes de ruído considerados como resíduo. Este modelo, segundo Serra, é particularmente eficiente para sons que contêm parciais inarmônicos ou componentes de ruídos importantes, o que acontece, pelo menos em parte, com a viola caipira. Por isso, adotamos neste trabalho uma perspectiva ampla do conceito de modelagem física, incluindo, além da síntese por delay lines, o modelo espectral de Serra, o modelo FOF e outros. Os algoritmos de modelagem foram projetados na linguagem de domínio público Csound, desenvolvida no MIT. Trata-se de um programa que permite analisar os problemas em um nível de detalhe aprofundado, de acordo com a demanda do projeto.

A diferença da modelagem física em relação aos outros métodos de síntese sonora digital é sua preocupação com uma relação de proximidade com os sons naturais. Em outras técnicas tradicionais de síntese, fundamentadas em propriedades matemáticas do som, há sempre o risco de que o som resultante soe artificial aos nossos ouvidos, com poucas semelhanças com os sons acústicos que nos parecem familiares. Isto pode tornar o som sintético pouco atraente à percepção humana e excessivamente conotado aos estereótipos da música eletrônica, remetendo nossas interpretações às trilhas de cinema de ficção científica, a estados alterados da mente ou simplesmente a certos gêneros de música dançante.

Outra vantagem de se estudar as respostas acústicas dos instrumentos reais, como no caso da viola caipira, é que seus sons têm uma rica variedade de espectros dinâmicos com evolução temporal. A evolução temporal interna dos espectros dinâmicos, em contraste com os de caráter estático de muitas sínteses clássicas, é que eles são interpretados pela nossa mente como tendo um sentido de vitalidade, de riqueza estética. Em outras palavras, esses sons nos parecem mais bonitos. Podemos fazer uma analogia entre as texturas de certos objetos feitos de plástico e outros feitos de materiais naturais: atribuímos valor maior a uma mala de couro natural do que a uma mala feita com um material sintético, mas, na medida em que o material artificial apresente uma textura rica em detalhes microscópicos elaborados (ainda que não prestemos muita atenção neles), aceitamos de bom grado a substituição. Por isso, a modelagem partiu da análise do espectro de amostras dos sons da viola caipira, eventualmente estáticas quando foram selecionadas apenas fragmentos das amostras, mas também outras mais longas, com caráter dinâmico, que nos permitiram compreender a evolução temporal do seu espectro.

Lembremos, entretanto, que a técnica de modelagem física privilegia as redes de filtros digitais e a retroalimentação dos ressonadores, de modo que a compreensão e a modelagem destes componentes permaneceram as preocupações centrais da pesquisa.

Salientemos, finalmente, que o propósito deste estudo não consistiu apenas em realizar a emulação realista do som da viola caipira, ainda que isso pudesse ser um resultado interessante. O objetivo principal foi estudar o mecanismo complexo de fun- 
cionamento desse instrumento, em que há uma interação dinâmica de diversas partes componentes para conceber um modelo abstrato de instrumento virtual que pudesse estender a geração de sonoridades para resultados inusitados para além das limitações físicas da construção e da execução dos instrumentos reais. Isso se coaduna com a concepção predominante na música contemporânea, especialmente na música eletroacústica, de que o design sonoro é inseparável da composição, ou seja, que, nesse gênero, forma e conteúdo são um todo indivisível.

\section{Filas de atraso (delay lines) na síntese Karplus-Strong}

A solução clássica da modelagem física deveria implicar o uso da equação geral da onda e considerar também a parte transitória, além da parte estável, do som produzido por um instrumento. Isso implicaria modelar muitos fatores do sistema vibratório, o que é difícil de implementar. Por isso, a modelagem física foi assimilada por uma solução alternativa baseada nos modelos de digital waveguide (guia de onda digital). Em sua implementação por linhas de atraso (SMITH III, 2010), o modelo torna-se mais intuitivo e computacionalmente eficiente (LOY, 2007, p.434).

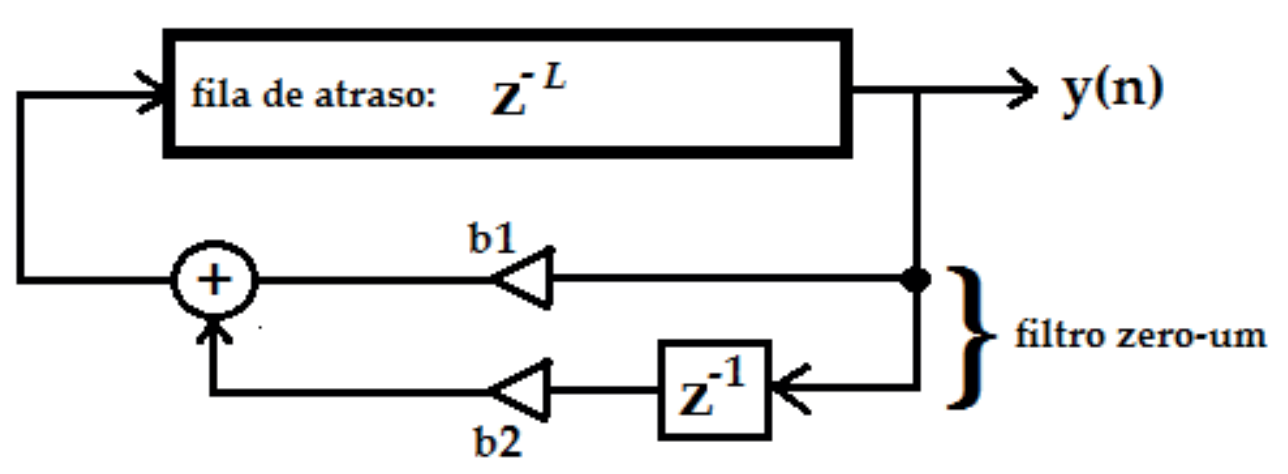

Fig. 2: Síntese de cordas pinçadas por fila de atraso (adaptado de LOY, 2007, p.435)

O funcionamento do modelo esquematizado na Figura 2 pode ser descrito, resumidamente, do seguinte modo: a linha de atraso é pré-carregada com uma sequência de números aleatórios; a parte inferior do diagrama representa a retroalimentação que tem dois multiplicadores redutores ou filtros zero-um cujos valores b1 e b2 podem ser ajustados entre zero e um; se $b 1=b 2=0$, ouvimos um pulso de ruído curto; se $b 2=0$ e b1=1, não há redução, e nossa percepção vai entender o sinal como um ruído com uma frequência dada pela razão entre a taxa de amostragem e o comprimento da fila; com valores intermediários como 1/2, há redução progressiva e ouviremos uma nota de timbre complexo, mas afinado, com decaimento exponencial. Esse modelo pode ser usado para simular instrumentos da família de cordas pinçadas como violões, harpas, pizzicatos de violinos e até tambores, dependendo do ajuste dos parâmetros. 
A literatura sobre esse tópico é extensa. Resenhá-la adequadamente está além do nosso escopo. Devemos, entretanto, observar sua implementação em Csound através da síntese Karplus-Strong (1983) na família do opcode "pluck" porque ele oferece um ponto de partida conveniente para nosso projeto.

O primeiro resultado a ser apresentado e discutido é a aproximação qualitativa ao som da viola caipira dos diversos opcodes de tipo pluck em Csound. Na Tabela 1 a seguir estão os opcodes com seus respectivos parâmetros, usados na aproximação qualitativa do som da viola caipira. O primeiro aspecto a ser considerado é o som metálico característico da viola. Os opcodes pluck e wgpluck produziram sons mais metálicos e, portanto, mais próximos da viola caipira do que repluck e wgpluck2, que não apresentaram este aspecto em nenhuma configuração de parâmetros.

No pluck, há a possibilidade de inserir uma maior quantidade de períodos de onda dentro da fundamental, pois icps determina o tamanho do buffer (quantidade de períodos a serem armazenados), e kcps determina o harmônico fundamental, partindo-se sempre das parciais inarmônicas geradas no ruído inicial. Como resultado, há um aumento da amplitude das parciais harmônicas de maior frequência, que simula adequadamente o brilho das cordas de metal do instrumento. O parâmetro ifilt exerce um papel similar, possibilitando o controle do brilho no timbre do instrumento. Portanto, o simples uso do opcode pluck do programa Csound já oferece uma alternativa simples para nosso projeto.

\begin{tabular}{|c|c|c|c|c|c|}
\hline Parâmetro & \multicolumn{4}{|c|}{ Opcode } & Descrição \\
\hline & pluck & repluck & wgpluck & wgphuck2 & \\
\hline kamp & 3000 & 12000 & - & 12000 & Amplitude \\
\hline iamp & - & - & 3000 & - & Amplitude \\
\hline kcps & p5 & - & - & - & frequência $(\mathrm{Hz})$ \\
\hline icps & $(\mathrm{p} 5) / 4$ & p5 & p5 & p5 & frequência $(\mathrm{Hz})$ \\
\hline idamp & - & - & 2 & - & abafamento/sustentação \\
\hline ifn & 0 & - & - & - & função de decaimento ciclico \\
\hline ifilt & - & - & 90 & - & Dim. duração de altas ou baixas freq. \\
\hline imeth & 6 & - & - & - & método de decaimento natural $(1, \ldots, 6)$ \\
\hline iplk & - & 0.95 & - & 0.95 & proporção da corda (0-1) tocada \\
\hline kpick & - & 0.2 & 0.1 & 0.2 & prop. corda $(0-1) \mathrm{p} /$ coleta da amostra \\
\hline krefl & - & 0.001 & - & 0.001 & coeficiente de reflexão $(0-1)$ \\
\hline axcite & - & $\begin{array}{r}\text { Oscil } \\
0,1,1\end{array}$ & $\begin{array}{c}\text { Oscil } \\
0,1,1\end{array}$ & - & sinal que excita a corda \\
\hline
\end{tabular}

Tab. 1: Opcodes de Csound para cordas pinçadas aplicados na síntese da viola caipira

Para o ensaio de simulação que desenvolvemos, procuramos reproduzir a importante característica das cordas duplas da viola caipira. Entretanto, é preciso esclarecer que esse instrumento comporta diversas afinações características, a saber: 


\begin{tabular}{|c|c|c|c|c|}
\hline & $\begin{array}{c}\text { Afinação } \\
\text { Natural }\end{array}$ & $\begin{array}{c}\text { Afinação } \\
\text { Cebolão em Mi }\end{array}$ & $\begin{array}{c}\text { Afinação } \\
\text { Cebolão em Ré }\end{array}$ & $\begin{array}{c}\text { Afinação } \\
\text { Rio Abaixo }\end{array}$ \\
\hline Corda 1 & Mi 4 & Mi 4 & Ré 4 & Ré 4 \\
\hline Corda 2 & Mi 4 & Mi 4 & Ré4 & Ré 4 \\
\hline Corda 3 & $\mathrm{Si} 3$ & $\mathrm{Si} 3$ & Lá 3 & $\mathrm{Si} 3$ \\
\hline Corda 4 & $\mathrm{Si} 3$ & $\mathrm{Si} 3$ & Lá 3 & $\mathrm{Si} 3$ \\
\hline Corda 5 & Sol 3 & Sol\# 3 & Fá\# 3 & Sol 3 \\
\hline Corda 6 & Sol 4 & Sol\# 4 & Fá\# 4 & Sol 4 \\
\hline Corda 7 & Ré 3 & Mi 3 & Ré 3 & Ré 3 \\
\hline Corda 8 & Ré4 & Mi 4 & Ré4 & Ré 4 \\
\hline Corda 9 & Lá 2 & $\mathrm{Si} 2$ & Lá 2 & Sol 2 \\
\hline Corda 10 & Lá 3 & $\mathrm{Si} 3$ & Lá 3 & Sol3 \\
\hline
\end{tabular}

Tab. 2: Afinações usuais da viola caipira

Para ampliar o controle e a compreensão da nossa simulação, continuamos a pesquisa procedendo uma adaptação de um opcode alternativo ao pluck projetado por Lazzarini et al. (2016, p.387), como descrito a seguir. Esse opcode String usa apenas as funções de defasagem delayr, delayw e deltap, além de implementar filtros e utilizar funções básicas da biblioteca Csound. 


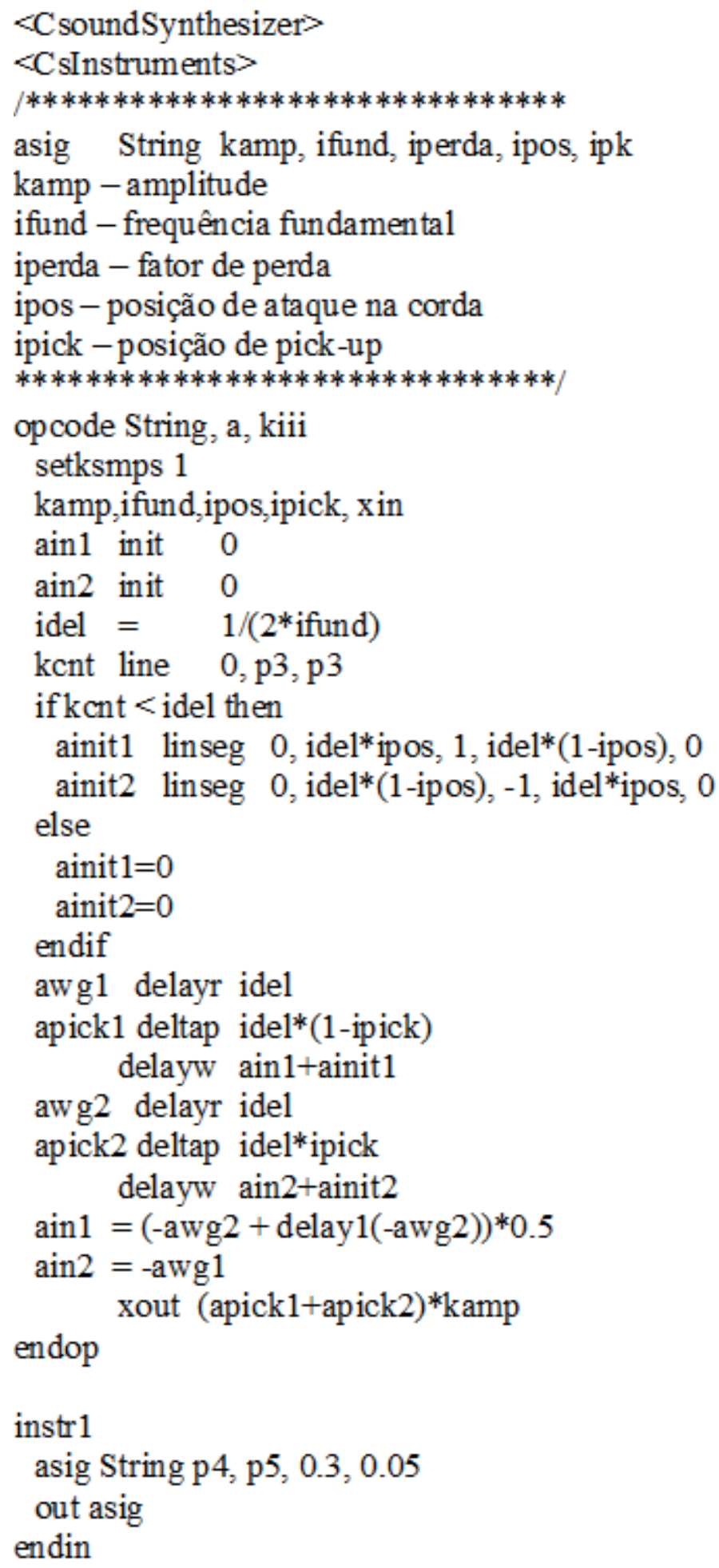

; arquivo score (partitura) de um arpejo das cordas soltas na afinação Cebolão em Mi ; adotamos um desvio de $2 \mathrm{~Hz}$ nas cordas duplas 8 e 10 para criar um efeito de batimento 


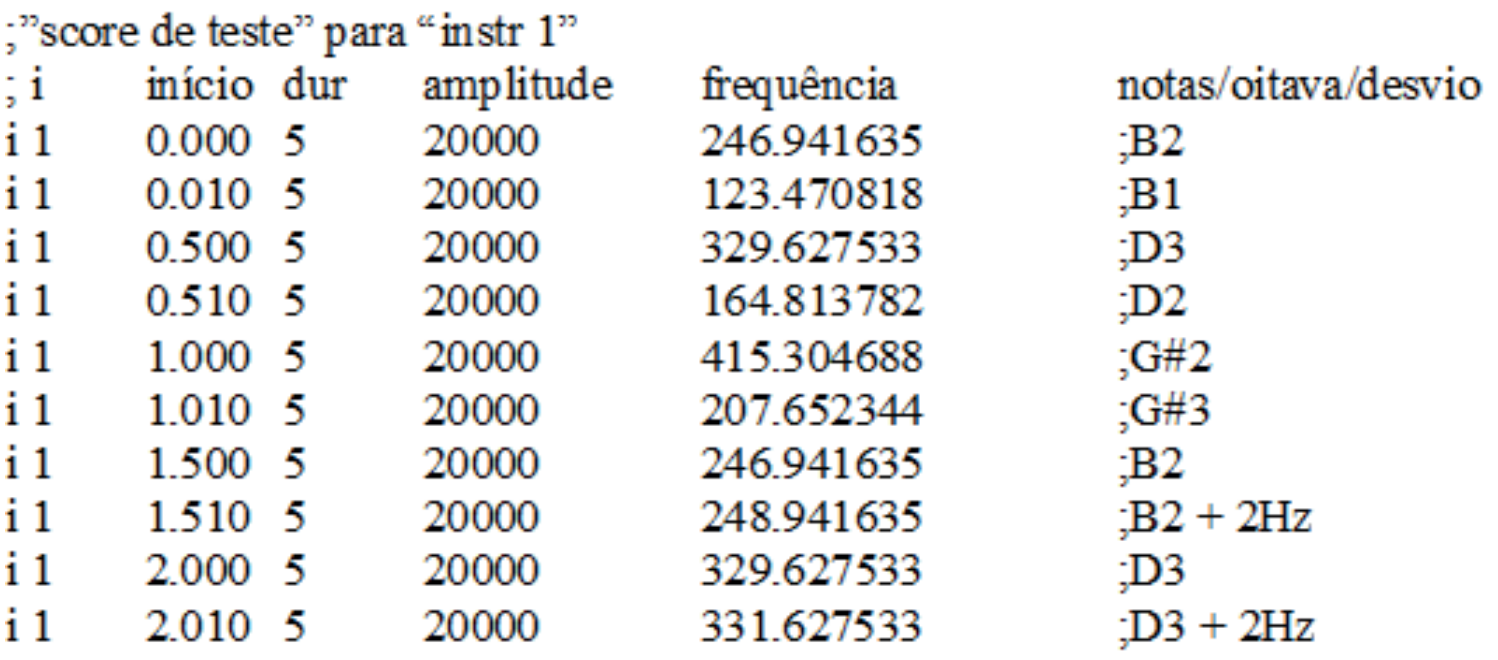

Cotejamos essa implementação com outro projeto desenvolvido por Mikelson (2000, p.370) para cordas graves. Como a tessitura da viola caipira não atinge esse registro, deixamos de lado os aspectos da proposta de Mikelson relacionadas a contornar as distorções que o processo de fila de atraso ocasiona em sons graves. Essa proposta é de fato uma implementação alternativa ao programa em $\mathrm{C}$ do opcode pluck, com a diferença de que podemos manipular aspectos internos dessa geração, resultando em menos limitações do que usar o pluck diretamente.

A Figura 3 apresenta uma versão simples do esquema da síntese Karplus-Strong.

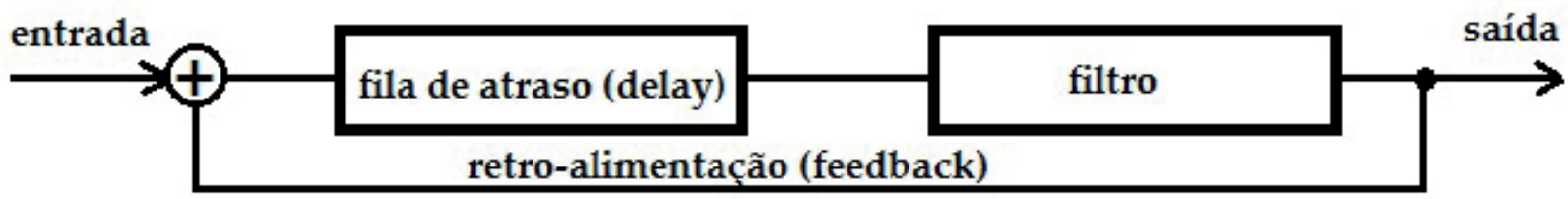

Fig. 3: Diagrama de bloco simplificado da síntese Karplus-Strong (adaptado de MIKELSON, 2000, p.371)

No processamento clássico de Karplus-Strong carrega-se a fila de atraso (delay line) com uma série de números aleatórios, que equivalem a um pulso de onda de ruído. Na proposta de Mikelson substitui-se esse impulso por uma onda triangular (ver kenvtriang no instr2), o que reduz a inarmonicidade do ataque. A seguir tira-se a média da amostra de saída atual com a amostra de saída anterior e adiciona-se essa média à entrada da fila de atraso. Esse é o processamento característico para simular uma waveguide.

Seja na solução clássica com números aleatórios, seja nesta de Mikelson com uma onda triangular, a fila de atraso é carregada com uma forma de onda com alta energia distribuída pelo espectro audível. $O$ artifício simples da média equivale a um filtro digital e simula o efeito de amortecimento causado pelo sistema mecânico de interação entre cordas e corpo do instrumento, especialmente no cavalete. 
;implementação devariante do modelo de Mikelson para simulação da viola caipira instr 2

\begin{tabular}{|c|c|c|c|}
\hline $\mathrm{qc}$ & $=$ & $\mathrm{p} 4$ & \\
\hline ipluck & $=$ & $1 /$ ifqc*p 5 & \\
\hline adlinha & init & 0 & \\
\hline abloq1 & init & 0 & \\
\hline abloq2 & init & 0 & \\
\hline afiltr & init & 0 & \\
\hline afeedback & init & 0 & \\
\hline koutenv & linseg & $0, .01,1, \mathrm{p} 3-0.11,1,0.1,0$ & ;envoltória da saída \\
\hline filtenv & linseg & $0,1.5,1,1.5,0$ & \\
\hline envoltóri & seguir & carrega a linha com uma onda tri & ular \\
\hline $\begin{array}{l}\text { kenvtriang } \\
\text { aenvstr } \\
\text { ainput }\end{array}$ & $\begin{array}{l}\text { linseg } \\
= \\
\text { tone }\end{array}$ & $\begin{array}{l}0 \text {, ipluck } / 4,-\mathrm{p} 5 / 2 \text {, ipluck } / 2, \mathrm{p} 5 / 2 \text {, } \\
\text { kenvtriang } \\
\text { aenvstr, } 1000\end{array}$ & luck/4, 0, p3-ipluck, 0 \\
\hline $\log 1$ & $=$ & afeedback-abloq $2+0.99 *$ abloq 1 & ;bloqueador de DC \\
\hline nha de ats & $\mathrm{ocom} r$ & retroalimentação filtrada & \\
\hline $\begin{array}{l}\text { adlinha } \\
\text { afiltr }\end{array}$ & $\begin{array}{l}\text { delay } \\
\text { tone }\end{array}$ & $\begin{array}{l}\text { abloq1*ainput, } 1 / \text { ifqc- } 15 / \mathrm{sr} \\
\text { adlinha. } 2000\end{array}$ & ;afinação corrigida \\
\hline orpo1 & reson & afiltr, 220,80 & ;ressonância da caixa \\
\hline orpo 1 & $=$ & acorpo $1 / 5000$ & \\
\hline orpo 2 & reson & afiltr, 140,40 & \\
\hline orpo 2 & $=$ & acorpo $2 / 20000$ & \\
\hline eedback & $=$ & afiltr & \\
\hline & & afeedback & \\
\hline & $\begin{array}{l}\text { out } \\
\text { endin }\end{array}$ & $50 *$ koutenv*(ao & (⿻) \\
\hline
\end{tabular}

O arquivo de partitura de teste para o "instr 2" pode ser o mesmo do "instr 1", porém devemos acrescentar valores para os parâmetros $p 5$ e p6, que controlam os efeitos descritos adiante. $O$ parâmetro $\mathrm{p} 6$ controla a duração do pulso inicial. Ao variar essa duração, produzimos simulações de diferentes tipos de ataque do pinçamento da corda.

No programa acima, Mikelson recomenda que se implemente uma correção de DC-offset que pode ser gerado pelo filtro e pelas condições iniciais, e que piora quando é retroalimentado. Por isso, há no programa acima a linha do filtro bloqueador de DC. A soma da amostra de entrada com o sinal DC bloqueado retroalimenta a fila de atraso com um comprimento que é o inverso da frequência. Esse resultado apresenta uma desafinação que é causada pelo atraso introduzido pelos filtros. Segundo Mikelson (2000, p.370), essa distorção pode ser corrigida subtraindo 15 amostras da linha de saída.

Além desses detalhes, note-se que o programa introduz efeitos de ressonância para simular a interação com o corpo do instrumento. Adotamos parâmetros diferentes dos sugeridos por Mikelson, baseados nas experiências de Paiva (2017), porque nosso instrumento é diferente do que Mikelson pretendeu emular. 


\section{Uso de dados experimentais na modelagem da viola caipira}

A literatura clássica sobre instrumentos musicais, como Fletcher (1998) e Henrique (2004), não trata diretamente da viola caipira mas aborda outros instrumentos da família, como o violão (ver FLETCHER, 1998, p.239-262) e o alaúde, entre outros instrumentos da mesma família (ver FLETCHER, 1998, p.264-269). Particularmente informativa é a análise que Fletcher apresenta sobre a contribuição das diferentes partes do violão para a formação do som do instrumento, como vemos na Figura 4. Esse esquema também é válido para a viola caipira ainda que as características da excitação das cordas e as proporções das interações (aliás, não mostrados na figura) sejam diferentes.

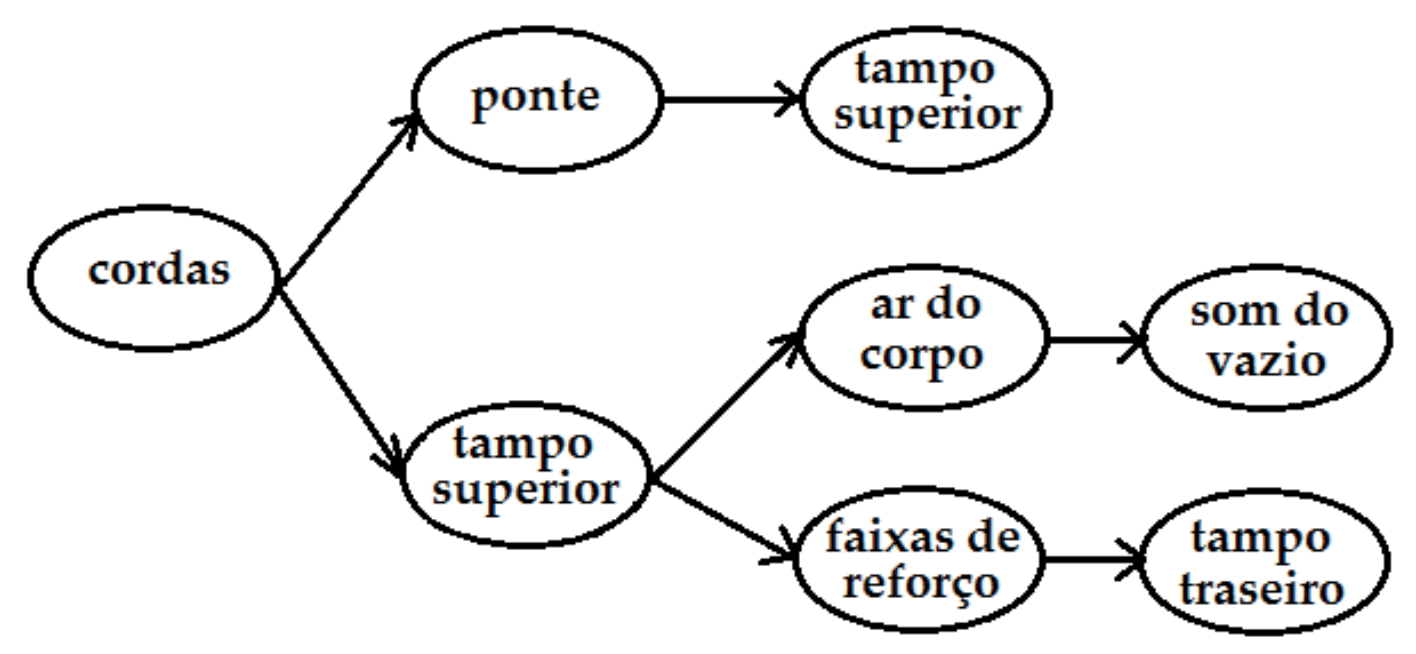

Fig. 4: Interação entre as partes componentes da viola (adaptado de FLETCHER, 1998, p.241)

Para uma aproximação mais fiel à viola caipira, nos baseamos nos dados levantados por Paiva (2017). que não são encontrados na literatura anterior. Paiva usou uma ampla gama de métodos para análise do instrumento, dentre elas observações da vibração das cordas com câmera de alta resolução, o que permitiu a observação de um fenômeno não tratado anteriormente na literatura: o choque de cordas dos pares oitavados quando tocados simultaneamente.

Além deste aspecto, Paiva observou que há ressonância e fenômenos de batimento quando se toca uma corda e as outras estão livres para vibrarem. Referindo-se à análise modal do tampo, o autor conclui que os quatro primeiros modos da viola caipira se assemelham ao do violão clássico, porém suas frequências são mais altas. Além disso,

a análise modal das curvas de mobilidade medidas na ponte viola caipira usando a Roving-Breaking Technique permitiu uma estimativa das frequências naturais, fatores de amortecimento e as formas das configurações dos modos de vibração nos pontos de acoplamento corda/cavalete usando uma análise modal de alta resolução. Tais resultados podem ser usados para alimentar modelos de síntese sonora (PAIVA, 2017, p. 96). 


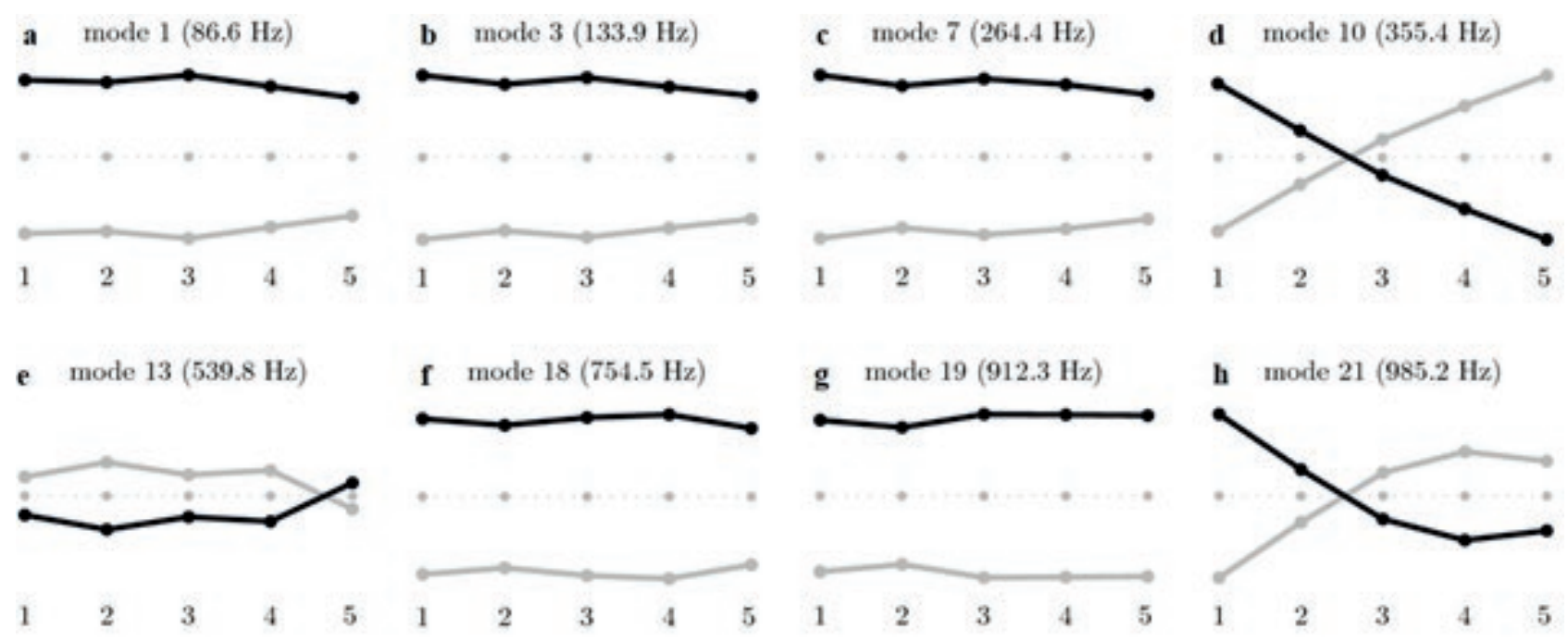

Fig. 5: Componentes modais na posição do cavalete (extraído de PAIVA, 2017, p.96)

Para simular os fenômenos descritos acima, usamos a estratégia de Serra (1997), da síntese com soma de componentes senoidais mais ruído de fundo. Observe-se que o efeito de choque entre os pares de cordas produz justamente ressonância por simpatia mais um ruído. Em trabalho anterior, usamos esse mesmo tipo de modelo para simular o som da kalimba (COELHO DE SOUZA, 2011). A diferença neste caso é que as relações não harmônicas da tecla da kalimba devem ser substituídas pelas relações harmônicas das cordas da viola caipira. Os efeitos dos formantes da viola caipira podem ser implementados com a técnica FOF descrita e implementada por Clarke (2000, p.294). Não vamos reproduzir os códigos em Csound dessas modelagens porque elas reproduzem, com pequenas adaptações, os códigos apresentados nos trabalhos citados.

\section{Bestiário III}

A composição de Bestiário III emprega uma versão flexibilizada do modelo clássico do tempo diferido na sincronização entre o violão e os sons eletrônicos. $O$ modelo clássico, de um playback contínuo em cima do qual o instrumentista executa seu instrumento em tempo real, foi usado reiteradamente pelos compositores do grupo da Columbia University desde a era dos sintetizadores analógicos. Particularmente Mario Davidovsky, com sua série dos doze Synchronisms para diversos instrumentos e sons eletrônicos, datando o primeiro de 1963 e o último de 2006, consagrou esse processo como viável e ao mesmo tempo desafiador para o instrumentista. O ciclo dos Bestiários utiliza um processo de divisão da parte eletrônica em diversos fragmentos que devem ser disparados em tempo real por um computador. Isso permite que o instrumentista tenha mais de flexibilidade na sincronização, permitindo desvincular o fraseado instrumental de uma precisão mecânica do tempo.

A composição utiliza uma série peculiar mostrada nas Figuras 6 e 7. Essa série é formada por dois hexacordes complementares que têm a mesma configuração 6-18 (012578), como demonstra a teoria dos conjuntos. Essa série permite, além da combi- 
natorialidade hexacordal, a utilização da relação de semelhanças de PCORD com módulo geracional ((11123)) entre os dois hexacordes (COELHO DE SOUZA, 2019, p.55). Entretanto, o processo de utilização da série não se limita a seguir o método dodecafônico clássico, nem os princípios do serialismo integral dos anos 1950-1960, mas se permite explorar a série como uma matriz geradora dos motivos que estão implícitos na sua própria estruturação. A página inicial da composição, mostrada na Figura 8 , permite que se reconheça o tipo de procedimento a que nos referimos.

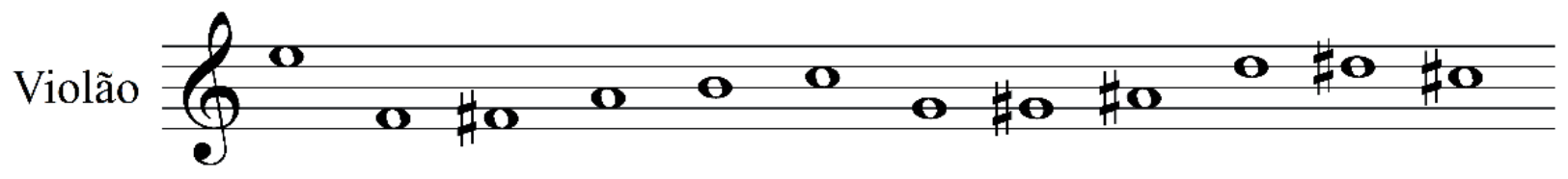

Fig. 6: Série utilizada em Bestiário III

\begin{tabular}{|c|c|c|c|c|c|c|c|c|c|c|c|}
\hline $\mathbf{C}$ & $\mathrm{C \#}$ & D & $\mathbf{F}$ & $\mathbf{G}$ & G\# & D\# & $\mathbf{E}$ & F\# & $\mathbf{A} \#$ & B & $\mathbf{A}$ \\
\hline B & $\mathbf{C}$ & C\# & $\mathbf{E}$ & F\# & $\mathbf{G}$ & D & D\# & $\mathbf{F}$ & $\mathbf{A}$ & $\mathbf{A} \#$ & $\mathbf{G} \#$ \\
\hline A\# & B & C & D\# & $\mathbf{F}$ & F\# & C\# & D & $\mathbf{E}$ & G\# & $\mathbf{A}$ & $\mathbf{G}$ \\
\hline $\mathbf{G}$ & G\# & $\mathbf{A}$ & $\mathbf{C}$ & D & D\# & $\mathbf{A \#}$ & B & C\# & $\mathbf{F}$ & F\# & $\mathbf{E}$ \\
\hline $\mathbf{F}$ & F\# & $\mathbf{G}$ & $\mathbf{A} \#$ & $\mathrm{C}$ & $\mathrm{C \#}$ & G\# & $\mathbf{A}$ & B & D\# & $\mathbf{E}$ & D \\
\hline $\mathbf{E}$ & $\mathbf{F}$ & F\# & $\mathbf{A}$ & B & C & $\mathbf{G}$ & G\# & $\mathbf{A \#}$ & D & D\# & C\# \\
\hline $\mathbf{A}$ & $\mathbf{A} \#$ & B & D & $\mathbf{E}$ & $\mathbf{F}$ & C & C\# & D\# & $\mathbf{G}$ & G\# & F\# \\
\hline G\# & $\mathbf{A}$ & $\mathbf{A} \#$ & C\# & D\# & $\mathbf{E}$ & B & $\mathbf{C}$ & D & F\# & $\mathbf{G}$ & $\mathbf{F}$ \\
\hline F\# & $\mathbf{G}$ & G\# & B & $\mathrm{C \#}$ & D & $\mathbf{A}$ & $\mathbf{A} \#$ & $\mathbf{C}$ & $\mathbf{E}$ & $\mathbf{F}$ & F\# \\
\hline D & D\# & $\mathbf{E}$ & $\mathbf{G}$ & $\mathbf{A}$ & $\mathbf{A \#}$ & $\mathbf{F}$ & F\# & G\# & $\mathbf{C}$ & C\# & B \\
\hline C\# & D & D\# & F\# & G\# & $\mathbf{A}$ & $\mathbf{E}$ & $\mathbf{F}$ & $\mathbf{G}$ & B & $\mathbf{C}$ & $\mathbf{A} \#$ \\
\hline D\# & $\mathbf{E}$ & $\mathbf{F}$ & G\# & $\mathbf{A} \#$ & B & F\# & $\mathbf{G}$ & $\mathbf{A}$ & $\mathrm{C} \#$ & D & C \\
\hline
\end{tabular}




\section{Bestiário III: Urutaurana}

(2019)

Rodolfo Coelho de Souza
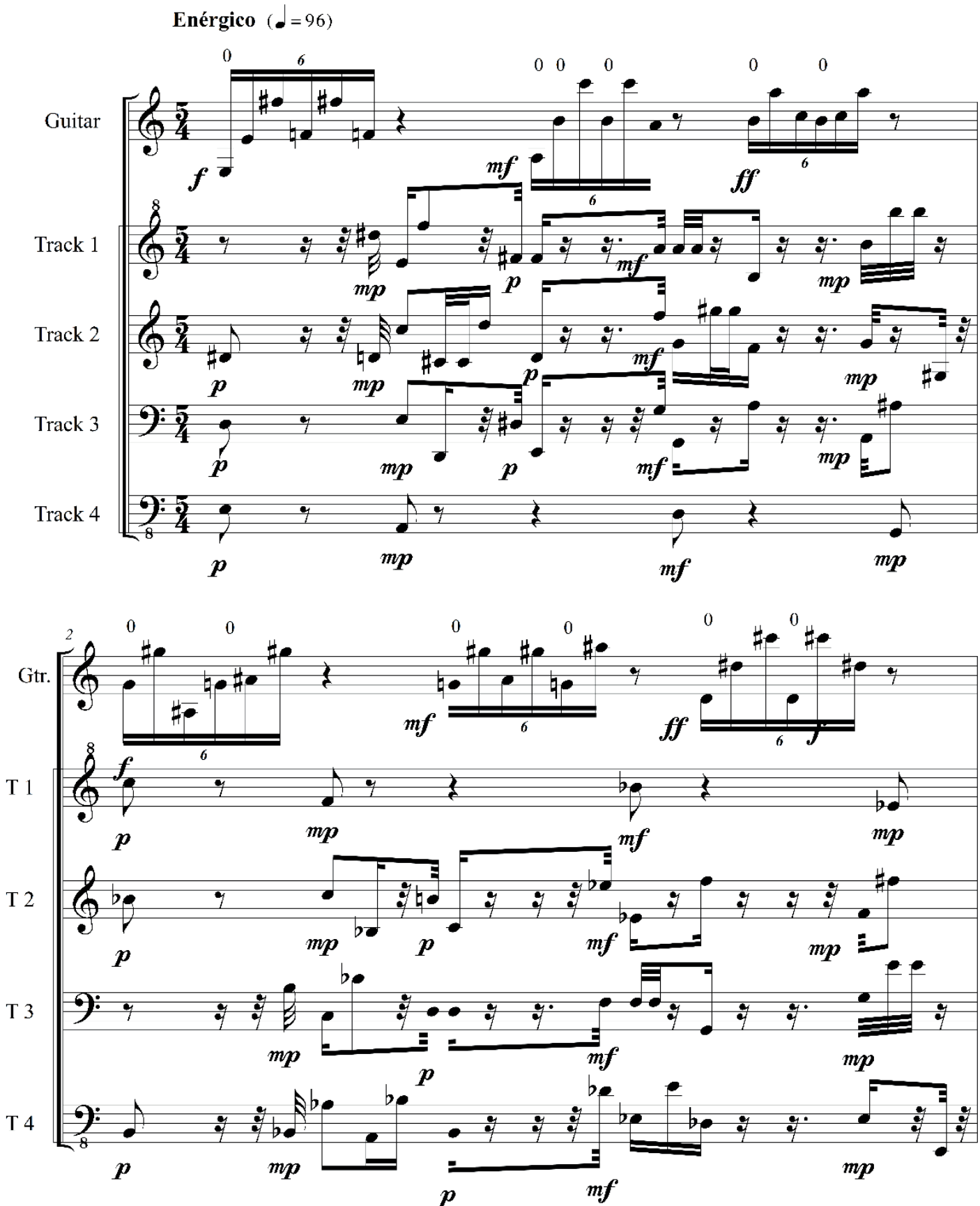

Fig. 8: Página inicial de Bestiário III 


\section{Conclusões}

A modelagem da síntese digital da viola caipira, usada como método composicional em Bestiário III para violão de sons eletrônicos, seguiu o preceito de Lazzarini (2016), que preconiza que a melhor maneira de se obter resultados de síntese sonora artisticamente convincentes é por meio da hibridização de soluções. Particularmente neste caso, em que o propósito não seria fazer uma reprodução realista da sonoridade do instrumento acústico, mas usar o estudo de suas propriedades para criar um instrumento virtual que criasse uma expansão acústica da sua sonoridade, a modelagem com diversos métodos mostrou-se a melhor alternativa. Esses métodos foram a síntese de Karplus-Strong, uma variante desse método proposto por Mikelson, a modelagem de soma de senoides mais ruído de Serra, e o reforço de formantes por FOF. Esses processos foram então somados em uma orquestra híbrida em que o peso variável dos diversos processos de síntese, ademais flexibilizados por uma ampla parametrização, resulta numa sonoridade de timbres complexos, justamente porque dinâmicos e variáveis, que não podem se materializar em nenhum instrumento acústico, especialmente com a velocidade de transição e coexistência de resultados que é possível no universo virtual.

Finalmente, ressaltamos que este estudo teve um duplo propósito. Por um lado, relatar uma pesquisa sobre waveguides e delay-lines correlacionadas a variações da síntese Karplus-Strong; por outro lado, mostrar que o timbre, como parâmetro fundamental na música contemporânea desde o início do século XX, e com repercussão crescente até o dia de hoje, não pode ser tratado analiticamente pela via taxonômica, dada a infinita variedade de resultados que podem ser gerados na síntese e na manipulação sonora, em que a maior parte dos sons resulta de multi-processamentos e combinações de processos. Para esse problema, há que se aplicar a proposta de Ferneyhough e Keller de usar a modelagem para se compreender a natureza dos resultados auditivos de uma peça, num processo que lembra o modelo da engenharia reversa, mas é diferente pois o propósito não é reproduzir o resultado original, mas encontrar um universo de aproximações que nos permita compreender, por meio da experimentação, a natureza de uma certa família de timbres.

\section{Referências}

COELHO DE SOUZA, R. The use of Brazilian folk instrument sounds in a concerto for computer and orchestra. Organised Sound. v. 10, p.31-36. 2005.

Um modelo de kalimba em Csound usado em O Livro dos

Sons. Música Hodie, v. 11, p.87-102. 2011.

Harmonic perception and voice leading spaces of set classes

related by unordered interval classes. Musica Theorica v. 3, n. 2, p.46-85. 2019. 
CLARKE, M. FOF and FOG Synthesis in Csound. In: BOULANGER, R. (ed.). The Csound Book: perspectives in software synthesis, sound design, signal processing and programming. Cambridge, MA: The MIT Press. 2000.

CORREAA, R. N. Viola caipira: das práticas populares à escritura da arte. Tese de doutorado em Musicologia. Escola de Comunicações e Artes. Universidade de São Paulo. São Paulo. 2014.

DE POLI, G.; ROCCHESSO, D. Physically based sound modelling. Organised Sound v. 3 n. 1, p.61-76. 1998.

FLETCHER, N; ROSSING, T. The Physics of Musical Instruments. 2 ed. New York: Springer. 1998.

HENRIQUE, L. L. Instrumentos Musicais. Lisboa: Fundação Calouste Gulbenkian. 2004.

KARPLUS, K.; STRONG, A. Digital synthesis of plucked string and drum timbres. Computer Music Journal, v. 7, n. 2. 1983.

KELLER, D.; FERNEYHOUGH, B. Analysis by modeling: Xenakis's ST/10-1 080262. Journal of New Music Research, v. 33, n. 2, p.161-171. 2004.

LAZZARINI, V; YI, S.; FFITCH, J.; HEINTZ, J.; BRANDTSEGG, O.; McCURDY, I. Csound: a sound and music computing system. Cham, Switzweland: Springer. 2016.

LOY, G. 2007. Musimathics: the mathematical foundations of music, v. 2. Cambridge, MA: The MIT Press. 2007.

MIKELSON, H. Mathematical modeling with Csound: from waveguides to caos. In: BOULANGER, R. (ed.). The Csound Book: perspectives in software synthesis, sound design, signal processing and programming. Cambridge, MA: The MIT Press. 2000.

PAIVA, G. O. Vibroacoustic characterization and sound synthesis of the viola caipira. Tese de doutorado - Faculdade de Engenharia Mecânica. UNICAMP. Campinas. 2017.

SERRA, X. Musical sound modeling with sinusoids plus noise. In: ROADS, C. (ed.). Musical Signal Processing. Lisse: Swets \& Zeitlinger. 1997.

SMITH III, J. O. Physical Audio Signal Processing: for virtual musical instruments and digital audio effects. Stanford: W3K Publishing. 2010. 